\title{
A New Fiber Optic Spring
}

\author{
S. YALTKAYA* \\ Akdeniz University, Faculty of Sciences, Department of Physics, 07058 Antalya, Turkey
}

\begin{abstract}
Optical sensors have attracted considerable attention in recent decades. Springs are used for a large variety of functions in industry including in sensor applications such as the measurement of force, acceleration, vibration, and pressure. In this study, a compression coiled spring and an optical fiber cable were combined to form a new optical fiber spring. The measurement part of the fiber optic spring was constructed by using a $3 \mathrm{~dB}$ fiber coupler and a fiber polarization beam splitter/combiner. During the study, a diode laser was used and silicon detectors measured the variations of the polarization state intensity. The spring constant of the optical fiber spring was obtained from decay of the free vibrations and Hooke's law. In conclusion, the new constructed fiber optic spring can be used in some industrial sensor applications.
\end{abstract}

DOI: $10.12693 /$ APhysPolA.125.635

PACS: 42.81.Pa, 07.10.Pz

\section{Introduction}

Optical sensors have attracted considerable attention in recent decades. Optical fiber sensors have been used in many industries, and research about optical fiber sensors has become a major field of endeavor. In addition, springs are used for a large variety of functions in sensor applications such as the measurement of force, acceleration, vibration, and pressure. Although they provide different information, coiled metal springs have been used for at least 500 years in a variety of machine design applications and mechanisms [1]. Hooke's law, which is widely used in physical modeling, states that the restoring force of a spring is directly proportional to a small displacement and to the spring constant $(k)$. Demonstration of the linear variation of displacement with applied force uses a fundamental experimental setup in physics laboratories.

In this study, a compression helical spring and an optical fiber cable were combined to form a new optical fiber spring (OFS). To our knowledge, this kind of design is absent in the literature. The fundamental principle of the OFS is to measure the two orthogonal states of polarization, during the back reflection of light. The back reflection takes place only in that part of the optical fiber located on the compression helical spring. Since this part of the optical fiber is the only part that moves with the spring, the variation in the polarization must occur in that part of the optical fiber.

The elasto-optic effect and the strain can be generated by applying force (and thereby the shear stress) to the optical fiber. There are two components of stress on any cross-section of coil of the helical spring and the optical fiber: a torsional shear stress due to the torque and a direct shear stress due to the force [2]. Under mechanical stress, the material of the optical fiber, especially the

\footnotetext{
*e-mail: yaltkaya@akdeniz.edu.tr
}

core and the cladding, become an anisotropic medium that may be completely characterized by the electric impermeability tensor. The index ellipsoid and hence the refractive indices for the optical wave travel in an arbitrary direction with arbitrary polarization in the optical fiber core [3]. Levels of variation of the anisotropic elasto-optic-induced refractive indices of the optical fiber core can be found in Bichler et al. work [4].

The optical anisotropy induced by torsion and bending has been studied theoretically by Tai and Rogowski [5] and similar discussions and calculations have been made in [6-8]. The stresses add and the maximum shear stress will occur in the inner part of the optical fiber, viz., the cross-section of the optical fiber's core. The maximum shear stress, $\tau_{\max }\left[\mathrm{N} / \mathrm{m}^{2}\right]$ is given by Eq. (1)

$$
\tau_{\max }=\frac{8 F D}{\pi d^{3}}+\frac{4 F}{\pi d^{2}}
$$

where $F$ is the applied force (load); $d$, wire diameter; $D$, mean coil diameter [2]. The maximum shear stress linearly increases with the force. Spring constant (rate), or force constant of the spring, a constant that depends on the spring's material (shear modules, $G\left[\mathrm{~N} / \mathrm{m}^{2}\right]$ ) and construction properties, is defined by:

$$
k=\frac{G d^{4}}{8 \pi D^{3}},
$$

where $n$ is the number of turns of the spring. In the case of only optical fiber, $G$ should be the shear modulus of the isotropic optical fiber.

Variations in the polarization state in the optical fiber, which is under the influence of force, were examined for use in some sensor applications [9-13].

\section{Experimental procedure}

A schematic diagram of the experimental setup is shown in Fig. 1. The laser beam, which is provided by a polarization-maintaining fiber pigtailed laser diode emitting at $660 \mathrm{~nm}$ wavelength $(10 \mathrm{~mW}$, Melles Griot), is coupled into a multimode optical fiber coupler $(3 \mathrm{~dB}, 2 \times 2$, Oz Optics Fused-12-IRVIS). In this work, a steel helical 
compression spring with squared ground ends was used. The free length of the spring, $D$, and $d$ were $3.5 \mathrm{~cm}$, $4 \mathrm{~cm}$ and $0.125 \mathrm{~cm}$, respectively. A part of one of the arms of the optical fiber coupler measuring $15 \mathrm{~cm}$ length and $125 \mu \mathrm{m}$ diameter was attached to the coils of the spring with glue. This part of the optical fiber acts as the sensing section. The tip of another fiber arm of the coupler was immersed into refractive index matching liquid (cedar oil, microscope grade) in order to get minimum back reflection. Also, the laser source light intensity was measured with a universal optical power meter (Melles Griot) via detector III under the glass beaker. The backscattering light from the sensing section was coupled into the entrance of a fiber polarization beam splitter (3 dB, Fitel DW902). After the separation of two orthogonally polarized laser beams, the beams were collected simultaneously with silicon photodiodes, detectors I and II. The spring was compressed at $0.45 \mathrm{~mm}$ intervals by the computer-controlled step motor and the polarized light intensities were measured via detectors I and II. The step motor's control unit was connected to the digital output channels and the rest of the connections were made via the analog inputs channel of an analog digital convertor (ADC, National Instruments PCI-6070E). The data acquisition used a LabVIEW program written for this application.

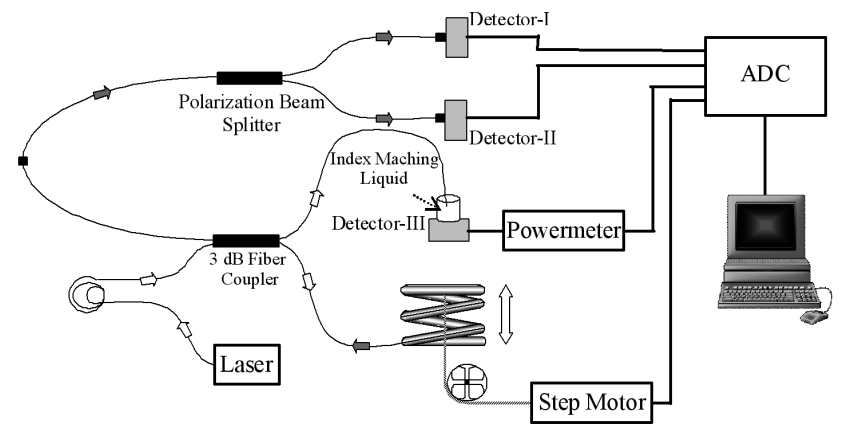

Fig. 1. Scheme of the experimental setup. See the text for details.

\section{Result and discussion}

In the first part of the work, the spring constant of the OFS was obtained by using Hooke's law

$$
\boldsymbol{F}=k \boldsymbol{x},
$$

where $x$ measures the displacement of the mass from an equilibrium position. To create the force, $10 \mathrm{~g}$ masses were utilized up to $100 \mathrm{~g}$. The spring constant was calculated using the slope of the force versus displacement graphic, as $98.1 \pm 3.6 \mathrm{~N} / \mathrm{m}$.

In the second part of the experiment, the spring constant was found from the decay of free vibration of the OFS with $100 \mathrm{~g}$ mass $(m)$ applied. During the free vibration of the OFS, the light intensities of detectors I and II were recorded as $I_{1}$ and $I_{2}$, respectively, taking an

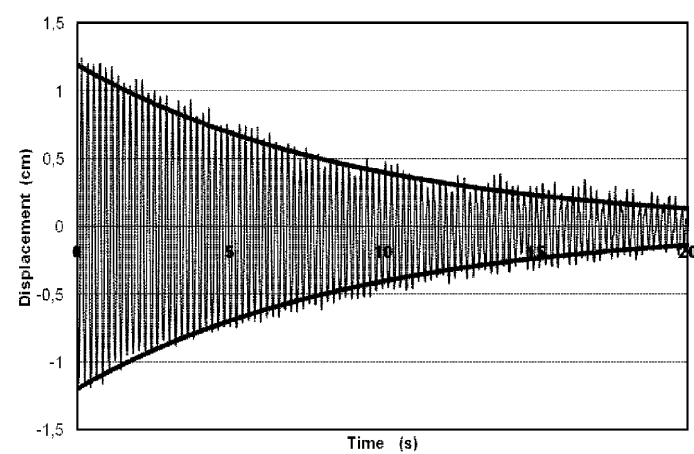

Fig. 2. The free vibrations of the fiber optic spring.

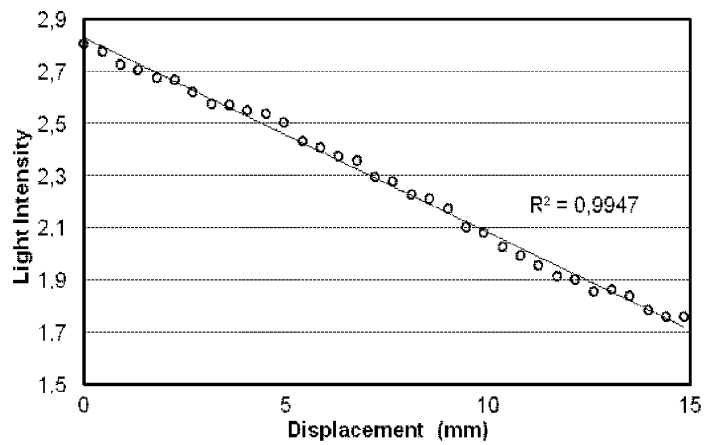

Fig. 3. The displacement of the FOS vs. light intensity $(I)$.

average of 50 data recordings every 0.01 s. From these, the variation of the light intensity was calculated using

$$
I=\frac{I_{1}+I_{2}}{I_{1}-I_{2}}
$$

and a graphic was drawn with $I$ versus time, as shown in Fig. 2, by using the calibration equation. The calibration equation was obtained from the variation of the intensity $(I)$ with the displacement in Fig. 3. To determine the frequency of the free vibration of the OFS, a Fourier analysis method was used with OriginPro software, giving an estimate of $5.04 \mathrm{~Hz}$ as shown in Fig. 4 . The spring constant of the OFS was calculated by using

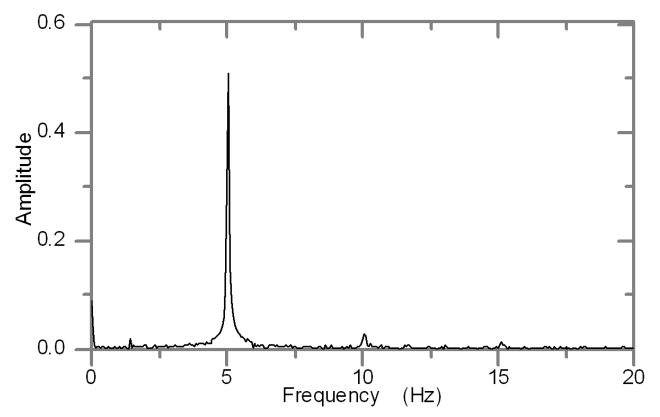

Fig. 4. Fast Fourier transform (FFT) analysis of the free vibration of the fiber optic spring. 
this frequency value with the aid of Eq. (5) [14]:

$$
\omega^{2}=\frac{k}{m}-\frac{b^{2}}{4 m^{2}},
$$

where $\omega$ is the angular frequency and $b$ is the damping coefficient. The damping coefficient was found from the envelope of the damped oscillatory curve that is also plotted in Fig. 2. The exponential envelope has the form $A \exp (-b t / 2 m)$ and the calculated $b$ value was $0.0218 \mathrm{~N} \mathrm{~s} / \mathrm{m}$. Finally, the spring constant was obtained as $100.2 \mathrm{~N} / \mathrm{m}$ by using Eq. (5).

In conclusion, the obtained spring constant from the vibration OFS is in good agreement with the spring constant derived using Hooke's law: $98.1 \pm 3.6 \mathrm{~N} / \mathrm{m}$. This new OFS can be used in some industrial fields and harsh conditions where other optical sensors cannot be used.

\section{References}

[1] V. Foley, S. Rowley, D.F. Cassidy, F.C. Logan, Technol. Culture 24, 399 (1983).

[2] P. Childs, Mechanical Design, Elsevier, Great Britain 2004, p. 231.
[3] B.E.A. Saleh, M.C. Teich, Fundamentals of Photonics, Wiley, USA 1991, p. 210.

[4] A. Bichler, S. Lecler, B. Serio, S. Fischer, P. Pfeiffer, J. Opt. Soc. Am. A 29, 2386 (2012).

[5] H. Tai, R. Rogowski, Opt. Fiber Technol. 8, 162 (2002).

[6] J.D. Mariani, G. Rodrigue, J. Opt. Soc. Am. B 23, 1743 (2006).

[7] A.M. Smith, Appl. Opt. 19, 2606 (1980).

[8] E. Suhir, J. Appl. Phys. 88, 3865 (2000).

[9] T. Okoshi, N. Fukaya, K. Kikuchi, Electron. Lett. 21, 895 (1985).

[10] S.C. Rashleigh, R. Ulrich, Opt. Lett. 5, 354 (1980).

[11] W. Eickhoff, Opt. Lett. 7, 629 (1982).

[12] I.P. Kaminow, IEEE J. Quant. Electron. QE-17, 15 (1981).

[13] V.U. Valiev, A.A. Simonov, D. Fu, D.R. Dzhuraev, J. Electromagn. Anal. Appl. 3, 128 (2011).

[14] A.P. French, Vibrations and Waves, W. Norton \& Co. Inc., New York 1971, p. 62. 